\title{
RELAÇÃO ENTRE INTENSIDADE DE DOR E CAPACIDADE FUNCIONAL EM INDIVÍDUOS OBESOS COM OSTEOARTRITE DE JOELHO
}

\author{
VASCONCELOS KSS ${ }^{1}$, DiAs JMD ${ }^{2}$, DiAs RC ${ }^{2}$ \\ ${ }^{1}$ Fisioterapeuta \\ 2 Departamento de Fisioterapia, Universidade Federal de Minas Gerais UFMG, Belo Horizonte, MG \\ Correspondência para: Karina Simone de Souza Vasconcelos, Rua Zenite, 470/101,Caiçara, CEP 30720-530, Belo \\ Horizonte, MG, e-mail: karinasimone@hotmail.com
}

Recebido: 03/05/2005 - Aceito: 07/12/2005

\begin{abstract}
RESUMO
Contextualização: A osteoartrite (OA) de joelho é uma doença crônica que acomete a cartilagem articular, provocando alterações ósseas, dor e rigidez à movimentação. A obesidade é um dos seus principais fatores de risco. As queixas de dificuldades funcionais são freqüentes, especialmente para a locomoção, que se torna mais lenta. Com os crescentes níveis de obesidade no Brasil, torna-se importante compreender como a OA de joelho afeta a capacidade funcional humana, a fim de se elaborar medidas de tratamento e prevenção. Objetivo: Analisar a influência da intensidade de dor, gravidade radiográfica, grau de obesidade e duração dos sintomas na capacidade funcional de indivíduos obesos com OA de joelho. Método: A intensidade de dor foi medida pelo questionário Western Ontario and McMaster Universities Index (WOMAC) e durante os testes funcionais. A gravidade radiográfica foi analisada pela classificação Kellgren-Lawrence e o grau de obesidade pelo índice de massa corporal (IMC). Foram realizados quatro testes funcionais de velocidade: marcha usual e rápida, subir e descer escadas. Resultados: A amostra foi constituída de 31 mulheres e 4 homens, com idade média de 51,65 \pm 10,72 anos e IMC médio de 41,16 $\pm 8,37 \mathrm{~kg} / \mathrm{m}^{2}$. As atividades em escadas estavam associadas a níveis mais intensos de dor. Apenas a intensidade de dor apresentou correlações significativas com os resultados nos testes de velocidade. Conclusão: A intensidade de dor é um fator que influencia a realização de atividades funcionais em indivíduos obesos com OA de joelho, mas outros estudos são necessários para identificar os fatores determinantes de capacidade funcional nesta população.
\end{abstract}

Palavras-chave: obesidade, osteoartrite de joelho, dor, capacidade funcional.

\section{ABSTRACT \\ Relationship Between Pain Intensity and Functional Capacity of Obese Individuals With Knee Osteoarthritis}

Background: Knee osteoarthritis is a chronic disease that affects joint cartilage, causing bone structure alterations, pain and stiffness during movement. Obesity is one of its principal risk factors. Complaints about functional disability are frequent, especially for locomotion activities, which people with knee osteoarthritis perform slower. With the increasing levels of obesity in Brazil, it is important to understand how knee osteoarthritis affects human functional capacity, in order to devise treatment and prevention measures. Objective: To examine the influence of pain intensity, radiographic severity, obesity level and symptom duration on the functional capacity of obese individuals with knee osteoarthritis. Method: Pain intensity was measured using the Western Ontario and McMaster Universities Index (WOMAC) questionnaire and during functional capacity tests. Radiographic severity was analyzed using the Kellgren-Lawrence classification and the obesity level was assessed using body mass index (BMI). Four functional velocity tests were performed: usual gait, fast gait, ascending stairs and descending stairs. Results: The sample consisted of 31 women and 4 men, with mean age of $51.65 \pm 10.72$ years and BMI of $41.16 \pm 8.37 \mathrm{~kg} / \mathrm{m}^{2}$. The greatest intensity of pain was reported during stair activities. Only pain intensity presented significant correlations with the results from the velocity tests. Conclusion: Pain intensity is a factor that influences functional activity performance among obese individuals with knee osteoarthritis. Further studies are necessary for identifying the determining factors for functional capacity in such populations.

Key words: obesity, knee osteoarthritis, pain, functional capacity. 


\section{INTRODUÇÃO}

A osteoartrite (OA) é uma doença crônica, caracterizada por degeneração da cartilagem articular, dor e rigidez à movimentação ${ }^{1}$. Estima-se que $4 \%$ da população brasileira apresente OA, sendo o joelho a segunda articulação mais acometida pela doença, com $37 \%$ dos casos ${ }^{2}$.

Um dos principais fatores de risco para esta doença é a obesidade ${ }^{3}$. A associação entre a OA de joelho e a obesidade também pode gerar maiores níveis de dor $^{4}$ e de dificuldades funcionais ${ }^{5}$, especialmente nas atividades de locomoção, que exigem movimentação e descarga de peso sobre as articulações afetadas.

Sabe-se que, no Brasil, o excesso de peso já supera os níveis de baixo peso em todas as regiões, sendo que $13 \%$ das mulheres e $8 \%$ dos homens no país são considerados obesos $^{6}$. Por isso, espera-se um aumento na prevalência e nos gastos da saúde pública com as comorbidades associadas à obesidade, como a OA de joelho. Por ser uma doença incapacitante, a OA de joelho gera gastos aumentados com tratamentos, além de uma maior necessidade de suporte social e familiar ${ }^{7}$, incluindo gastos com a Previdência Social ${ }^{8}$.

Uma das formas de se avaliar os prejuízos funcionais associados à OA de joelho são os questionários, no qual os indivíduos relatam suas dificuldades. Devido à sua especificidade para a OA de joelho, o questionário Western Ontario and McMaster Universities Osteoarthritis Index (WOMAC) ${ }^{9}$ é um questionário amplamente recomendado para esse fim. Indivíduos com OA de joelho também apresentam alterações funcionais objetivas, sendo mais comuns a menor velocidade para marcha ${ }^{10} \mathrm{e}$ para subir ou descer escadas ${ }^{11}$. Por isso, testes de velocidade são considerados métodos confiáveis e simples, com baixos custos, para avaliar estas alterações objetivas ${ }^{12}$.

Além da obesidade, diversos fatores podem influenciar a capacidade funcional em indivíduos com OA de joelho, entre eles a gravidade da doença ${ }^{13}$, a amplitude de movimento e a força muscular ${ }^{14}$, a idade e o sexo ${ }^{15}$. A intensidade de dor é um fator determinante para o relato de dificuldades ${ }^{16} \mathrm{e}$ pode afetar as velocidades usual e rápida de marcha ${ }^{17}$.

A compreensão do processo de alterações funcionais na OA de joelho pode contribuir para a elaboração de medidas de prevenção e tratamento em populações de risco como os obesos. Este estudo se propôs a analisar como a intensidade da dor pode influenciar a capacidade funcional objetiva de indivíduos obesos com OA de joelho, em um delineamento transversal e observacional. Também foi analisada a influência da gravidade dos achados radiográficos, da idade, índice de massa corporal (IMC) e tempo dos sintomas de osteoartrite na capacidade funcional objetiva.

\section{METODOLOGIA}

\section{Aprovação}

Esta pesquisa foi aprovada pelo Comitê de Ética em Pesquisa da Universidade, parecer $n^{\circ}$ ETIC 222/04 de acordo com a Resolução 196/96 do Conselho Nacional de Saúde. Todos os voluntários assinaram e receberam uma cópia do termo de consentimento livre e esclarecido.

\section{Amostra}

A amostra foi selecionada por conveniência, com voluntários participantes de ambulatórios de tratamento de obesidade ou de serviços de reabilitação de Hospitais Universitários e da Santa Casa de Misericórdia da cidade. Estes participantes eram encaminhados aos pesquisadores responsáveis, no Laboratório de Desempenho Motor e Funcional Humano da Universidade, por profissionais de saúde que os atendiam e que estavam cientes das condições deste estudo. Uma vez verificados os critérios de inclusão e exclusão, os indivíduos encaminhados eram convidados a participar deste estudo como voluntários.

Foi utilizado como base para a análise estatística do cálculo amostral, o desvio-padrão estimado da variável de interesse, isto é, a capacidade funcional objetiva, a partir de estudos que avaliaram medidas similares de velocidade de atividades em escadas ${ }^{11}$ e de marcha ${ }^{12}$, com indivíduos com osteoartrite de joelho. A seguinte fórmula foi utilizada: $n=(z \alpha / 2 \times \partial / \mathrm{d})^{2}$. Onde: $\mathbf{n}$ é o tamanho da amostra, $\mathbf{Z}_{\alpha / 2}$ é o valor da ordenada da distribuição que deixa abaixo dele área igual a $\alpha / 2$, $\partial$ é o desvio-padrão da variável de interesse e $\mathbf{d}$ é tal que a probabilidade de um desvio d entre a média da variável de interesse (obtida no estudo) e a média real seja menor ou igual a $\boldsymbol{\alpha}$. Determinou-se em 0,05 ( $\boldsymbol{\alpha})$ esta probabilidade de erro, considerando-se aceitável um erro (d) menor que 1/3 de um desvio-padrão ( $\partial$ ).

Com base nestes cálculos, foram incluídos 35 voluntários de ambos os sexos, com Índice de Massa Corporal (IMC) $>30 \mathrm{~kg} / \mathrm{m}^{2}$, com o diagnóstico clínico e radiográfico de OA de joelho segundo os critérios de Altman et al. ${ }^{1}$. Todos apresentavam marcha independente. Foram excluídos indivíduos que apresentassem: dor no joelho ou incapacidade funcional referente a esta articulação devido a lesões ligamentares, meniscais ou musculares diversas à $\mathrm{OA}$; prótese total ou parcial em um ou ambos os joelhos ou quadris; artrite reumatóide, fibromialgia, lúpus eritematoso sistêmico e doenças reumáticas sistêmicas; cardiopatias descompensadas; déficits auditivos ou visuais que impedissem a realização dos testes e medidas; agudização da OA de joelho; doenças neurológicas que afetassem a locomoção.

Uma vez que a OA de joelho é uma doença crônica, alguns voluntários faziam uso de analgésicos ou antiinflamatórios. Sendo observacional, o objetivo deste estudo 
foi retratar a capacidade funcional o mais próximo da realidade do dia-a-dia. Desta forma, não foi recomendada a interrupção do uso deste tipo de medicamentos.

\section{Dor}

O questionário WOMAC foi aplicado na sua versão traduzida e validada para o Brasil ${ }^{18}$ por meio de entrevista assistida. A intensidade de dor foi representada pelos escores na seção de dor do questionário, nos níveis: nenhuma, pouca, moderada, intensa e muito intensa. A mesma escala foi utilizada para mensurar a dor durante a realização dos testes de capacidade funcional. Para análise dos dados, estes níveis foram transformados respectivamente em 0, 25, 50, 75 e 100.

\section{Gravidade radiográfica}

Para avaliar a gravidade da OA de joelho através dos critérios Kellgren-Lawrence ${ }^{19}$, foram examinadas radiografias da articulação tibiofemural em imagens ântero-posteriores, com carga sobre os membros inferiores. Cada radiografia foi classificada por um examinador que desconhecia o estado clínico dos voluntários em: grau I = provável diminuição do espaço articular, com possível osteofitose; grau II = osteófitos bem definidos e possível diminuição do espaço articular; grau III = múltiplos osteófitos, clara diminuição do espaço articular e possíveis deformidades nas extremidades ósseas; grau IV = grandes osteófitos, intensa diminuição do espaço articular, esclerose grave e extremidades ósseas com deformidades definidas $^{19}$.

\section{Testes de capacidade funcional}

A capacidade funcional objetiva foi avaliada por quatro testes de velocidade: marcha usual, marcha rápida, subir escadas e descer escadas.

Os testes de marcha foram realizados em um corredor plano e sem obstáculos, com distância percorrida de 25 m. Os três primeiros e os dois últimos metros foram desprezados como aceleração e desaceleração. Para medir a velocidade foram utilizadas células fotoelétricas (Kit Multisprint ${ }^{\circledR}$, Inserra Indústria Mecânica LTDA, Belo Horizonte, Minas Gerais). A figura 1 mostra o Kit Multisprint ${ }^{\circledR}$. Para familiarização, os voluntários percorreram o corredor uma vez. Para o teste de marcha usual, eram orientados a "caminhar em um ritmo normal”. Para a marcha rápida, a "caminhar o mais rápido possível”, com o estímulo verbal "rápido, rápido”.

Os outros testes foram realizados em uma escada comum de prédio, com corrimão. Para familiarização, os voluntários subiram e desceram os seis degraus uma vez. Para os testes, eles foram instruídos a realizar a atividade “em um ritmo normal, da maneira como tivessem costume de fazer no dia-a-dia”, sendo permitido o uso do corrimão. Também era permitido realizar os testes colocando os dois pés em cada degrau, ou alternando o apoio com cada pé em um degrau. O mesmo padrão quanto ao uso do corrimão e o tipo de apoio eram utilizados durante todo o teste.

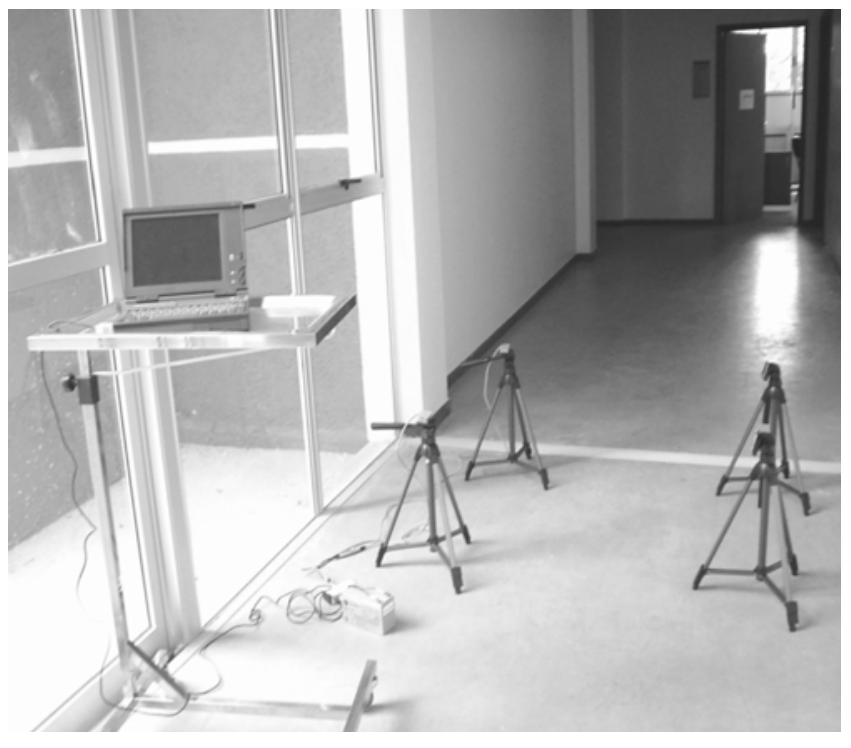

Figura 1. Kit para Avaliação Computadorizada de Rendimento Físico Multisprint ${ }^{\circledR}$, utilizado para avaliar as velocidades de marcha usual e rápida.

Os voluntários iniciavam os testes ao comando “já” do examinador, momento em que era disparado o cronômetro. A contagem de tempo era interrompida quando se alcançava o último degrau com os dois pés, sempre olhando para frente.

A ordem dos testes foi aleatorizada por sorteio e os voluntários foram instruídos a utilizar um calçado usual, que considerassem confortável e seguro. Foram realizadas três medidas para cada teste, com intervalo de 15 a 30 segundos entre cada medida e 30 a 60 segundos entre cada tipo de teste. A coleta de dados foi realizada em uma única sessão, entre às 14 e 17 horas.

\section{Análise estatística}

Os dados foram analisados com o software SPSS Stastitical Package for Social Sciences (versão 11.5). Além das análises descritivas, foi utilizado o teste de correlação não-paramétrico com o coeficiente de Spearman para avaliar a relação da intensidade de dor e gravidade radiográfica com a capacidade funcional. Para a relação da idade e obesidade com a capacidade funcional foi utilizado o teste paramétrico com o coeficiente de correlação linear de Pearson. O nível de significância foi estabelecido como $\alpha=0,05$.

\section{RESULTADOS}

\section{Amostra}

A amostra foi constituída por 31 mulheres e 4 homens. A idade média foi de 51,65 \pm 10,72 anos (mínimo de 29,00 e máximo de 67,83). A altura média dos voluntários foi de $1,57 \pm 0,092$ metros $(\mathrm{m})$ e o peso médio foi de $102,30 \pm$ 25,037 quilos (Kg). O IMC médio foi de 41,16 \pm 8,37 kg/ $\mathrm{m}^{2}$. 
Apenas um voluntário apresentava acometimento unilateral da OA de joelho. O tempo médio de início dos sintomas da OA de joelho foi de 6,47 \pm 4,16 anos.

\section{Dor}

A média do escore total da seção de dor do WOMAC foi de 42,85 $\pm 17,83$. O item de dor caminhando no plano apresentou valor médio de $33,57 \pm 28,40$ e o item de intensidade de dor subindo ou descendo escadas de 62,14 $\pm 22,98$. A tabela 1 apresenta os níveis médios de dor durante cada teste de capacidade funcional.

Tabela 1. Níveis de dor durante os testes de capacidade funcional.

\begin{tabular}{cc}
\hline TESTE & ${\text { MÉDIA } \pm \text { DP }^{\dagger}}^{{ }^{\dagger}}$ \\
\hline Marcha usual & $20,71 \pm 25,35$ \\
Marcha rápida & $26,42 \pm 27,74$ \\
Subir escadas & $32,14 \pm 24,68$ \\
Descer escadas & $37,14 \pm 26,68$ \\
\hline
\end{tabular}

${ }^{\dagger} \mathrm{DP}=$ desvio-padrão

\section{Classificação Radiográfica}

Para a avaliação radiográfica da OA de joelho, obtivemos 33 radiografias. Em caso de discrepância entre os dois joelhos acometidos pela OA, foi considerado o resultado da articulação com maior grau. A maioria dos voluntários apresentou grau II (11 voluntários) e III (13 voluntários).

\section{Capacidade Funcional}

A velocidade média de marcha usual foi de 1,13 $\pm 0,21$ $\mathrm{m} / \mathrm{s}$ e a de marcha rápida de $1,53 \pm 0,25 \mathrm{~m} / \mathrm{s}$. Para subir escadas, a velocidade média do grupo foi de $0,97 \pm 0,29$ degraus/s e para descer escadas de 1,02 $\pm 0,35$ degraus/s.

\section{Relação entre intensidade de dor e capacidade funcional}

O escore total da seção de dor do WOMAC não apresentou correlação estatisticamente significante com a velocidade de marcha usual $(\mathrm{r}=-0,180, \mathrm{p}=0,3)$, nem com a velocidade de subir escadas $(\mathrm{r}=-0,32, \mathrm{p}=0,058)$. Para a velocidade de marcha rápida e de descer escadas, foram observadas correlações estatisticamente significantes e inversas $(r=-0,399, p=0,018$ e $r=-0,379, p=0,025$, respectivamente).

A intensidade de dor no item caminhando no plano estava correlacionada inversamente com a velocidade de marcha usual $(\mathrm{r}=-0,411, \mathrm{p}=0,014)$ e de marcha rápida $(\mathrm{r}=-0,591$, $\mathrm{p}=0,001)$, ambas estatisticamente significantes. Para o item subindo ou descendo escadas, não houve correlação significante entre intensidade de dor e velocidade de subir escadas $(r=-0,308, p=0,072)$, ao contrário da velocidade de descer escadas $(r=-0,346, p=0,042)$.

Para a intensidade de dor durante os testes, foram observadas correlações inversas, estatisticamente significantes, com a velocidade de marcha usual $(r=-0,351, p=0,039)$, de marcha rápida $(\mathrm{r}=-0,347, \mathrm{p}=0,041)$ e para subir escadas $(r=-0,362, p=0,033)$. Não houve correlação significante para o teste de descer escadas $(r=-0,261, p=0,130)$.

\section{Relação entre capacidade funcional e outras variáveis}

Não foram encontradas correlações estatisticamente significantes entre os resultados dos testes de velocidade e as de gravidade radiográfica, idade, IMC e tempo dos sintomas da OA de joelho.

\section{DISCUSSÃO}

Este estudo procurou compreender como a intensidade de dor influencia a capacidade funcional de indivíduos obesos com OA de joelho. Ambas as variáveis são de grande relevância clínica, uma vez que os principais alvos do tratamento para as pessoas que sofrem com a OA de joelho constituem a redução dos níveis de dor e a melhora na capacidade de realização das atividades funcionais.

Em termos clínicos e radiográficos, pode-se dizer que a amostra apresentava um acometimento moderado da OA de joelho. Os resultados quanto à intensidade de dor mostraram altos valores de desvio-padrão, demonstrando a grande variação deste sintoma entre indivíduos com OA de joelho. Os maiores níveis de dor associados às atividades em escadas, tanto no questionário WOMAC quanto na realização dos testes pode refletir o maior impacto na articulação do joelho que ocorre durante estas atividades, em relação à marcha no plano ${ }^{20}$.

Os resultados deste estudo demonstraram correlações estatisticamente significantes entre intensidade de dor e capacidade funcional, apesar de não ter sido controlada a variável referente ao uso de medicamentos. De maneira geral, foram encontradas correlações de fraca a moderada intensidade, de forma semelhante aos outros estudos ${ }^{21,22}$.

O escore da seção de dor do questionário WOMAC apresentou correlação significativa apenas com as atividades de marcha rápida e descer escadas. Provavelmente estas duas atividades representam maiores desafios à capacidade funcional de obesos com OA de joelho. A marcha rápida, em especial, não era uma atividade comum no dia-a-dia dos voluntários deste estudo.

Já analisando o nível de dor no item caminhando no plano, foram observadas correlações moderadas tanto com a velocidade de marcha usual quanto com a velocidade de marcha rápida. Estes resultados demonstram a especificidade da dor na OA de joelho e a aplicabilidade do questionário WOMAC em analisá-la, considerando diferentes atividades funcionais. 
Quanto ao nível de dor no item subindo ou descendo escadas, houve correlação significativa apenas com a velocidade de descer escadas, o que pode refletir o tipo de pergunta deste item, que agrega duas atividades: subir $e$ descer escadas. Para muitos dos voluntários, a atividade de descer escadas era especialmente mais dolorosa do que a de subir escadas. Ao responder o questionário, é provável que tenham associado o maior nível de dor àquela atividade.

Para a intensidade de dor durante os testes de capacidade funcional, não houve correlação significativa apenas para a velocidade de descer escadas, diferentemente da relação entre a dor medida pelo questionário WOMAC e a velocidade para descer escadas. A variação da dor na OA de joelho de acordo com a atividade funcional realizada pode explicar este contraste. Ao responder um questionário sobre dor ao descer escadas, o voluntário vai se reportar às escadas que utiliza no dia-a-dia, que variam em largura, extensão, forma e altura dos degraus, disponibilidade de corrimão, etc. Além disso, as demandas de uma mesma atividade podem variar. Pode ser preciso descer vários lances de degraus para alcançar rapidamente um ônibus, ou descer calmamente três degraus na porta de casa. Um teste de capacidade funcional é realizado em um ambiente padronizado com demandas específicas, que pode ou não ser mais desafiador que as atividades funcionais do dia-a-dia. É possível que o teste de descer escadas deste estudo fosse menos desafiador que as atividades funcionais dos voluntários, uma vez que era permitido que o realizassem à sua maneira usual. A única demanda imposta era chegar ao final da escada. Não havia exigências de tempo, performance ou outros objetivos. Alguns indivíduos relataram que poderiam realizar os testes com mais velocidade ou sem a ajuda do corrimão, se solicitados, apesar da possibilidade de sentirem dor mais intensa. Isto demonstra uma capacidade de atender a maiores demandas funcionais, apesar de um estímulo negativo como a dor. Estudos demonstram que indivíduos com OA de joelho, entre eles os obesos, são capazes de modificar estratégias de movimento para reduzir a dor e o estresse articular ${ }^{23,24}$. É possível, que, nas condições do estudo, os voluntários tenham se adaptado à tarefa de descer escadas, reduzindo a dor e sua influência na velocidade com que realizavam a atividade. Por outro lado, o teste de subir escadas parece ter alcançado as demandas funcionais que são influenciadas pelo nível de dor entre indivíduos obesos com OA de joelho.

Como diversas estruturas contribuem para o funcionamento da articulação do joelho e as repercussões da OA de joelho não se restringem à parte óssea da articulação, era provável o resultado quanto à ausência de correlação entre a gravidade radiográfica e as medidas de capacidade funcional, como observado por outros estudos ${ }^{25,26}$. A incidência radiográfica em perfil não é considerada como mandatória para a classificação de gravidade radiográfica de Kellgren-Lawerence ${ }^{19}$, mas é possível que contribuísse de outra forma na influência sobre a capacidade funcional, apre- sentando, por exemplo, correlação entre o estado da articulação fêmuro-patelar e as amplitudes de flexão realizadas durante as atividades em escadas.

Da mesma forma, o tempo dos sintomas, considerado isoladamente como um dos fatores de gravidade OA de joelho da doença, pode não representar a diversidade entre a progressão e a capacidade de adaptação entre os indivíduos com a doença.

Também é importante lembrar que o IMC representa apenas uma alteração no equilíbrio energético do indivíduo, não permitindo análises de alterações metabólicas ou composições de massa gorda ou magra. É possível que estes fatores não englobados pelo IMC tenham maior repercussão na capacidade funcional de indivíduos obesos.

Este estudo apresentou considerações quanto a obesos com OA de joelho e seus resultados não podem ser generalizados para outros grupos com esta doença. O tamanho reduzido da amostra e os altos valores de desvio-padrão podem ter influenciado a ausência de relação entre algumas variáveis e a capacidade funcional, achados que não podem ser tomados como definitivos. Além disso, a velocidade representa apenas um dos aspectos referentes à realização de atividades funcionais como a marcha e o subir ou descer escadas. A capacidade funcional humana é complexa e abrangente, envolvendo diversas outras características além do tempo despendido para a realização de uma determinada atividade ${ }^{27}$.

\section{CONCLUSÃO}

Pode-se concluir que a intensidade de dor está relacionada à capacidade funcional objetiva de indivíduos obesos com OA de joelho, de forma moderada. Como a dor, isoladamente, não é capaz de explicar as alterações e prejuízos funcionais associados à OA de joelho, sua redução permanece como objetivo clínico importante, mas confirmam-se as evidências de que outros recursos de tratamento são necessários para minimizar os efeitos deletérios da OA de joelho na capacidade funcional e potencializar o uso e a proteção da articulação afetada.

\section{REFERÊNCIAS BIBLIOGRÁFICAS}

1. Altman R, Asch E, Bloch D, Bole G, Borenstein D, Brandt K, et al. Development of criteria for the classification and reporting of osteoarthritis. Classification of osteoarthritis of the knee. Arthritis Rheum 1986; 29 (8): 1039-49.

2. Senna ER, Barros ALP, Silva EO, Costa IF, Pereira LVB, Ciconelli RM, et al. Prevalence of rheumatic diseases in Brazil: A study using the COPCORD approach. J Rheumatol 2004; 31 (3): 5947.

3. Felson DT, Anderson JJ, Naimark A, Walker AM, Meenan RF. Obesity and knee osteoarthritis: The Framingham Study. Ann Int Med 1988; 109:18-24. 
4. Jinks C, Jordan K, Croft P. Measuring the population impact of knee pain and disability with the Western Ontario and McMaster Universities Osteoarthtis Index (WOMAC). Pain 2002; 100(1-2): 55-64.

5. Ettinger WH, Davis MA, Neuhaus JM, Mallon KP. Long-term physical functioning in persons with knee osteoarthritis from NHANES I: effects of comorbid medical conditions. J Clin Epidemiol 1994; 47: 809-15.

6. IBGE - Instituto Brasileiro de Geografia e Estatística. Pesquisa de Orçamentos Familiares - POF 2002-2003; 2004.

7. Lapsley LM, March LM, Tribe K, Cross MJ, Brooks, PM. Living with osteoarthritis: patient expenditures, health status and social impact. Arthritis Rheum 2001; 45(3): 301-6.

8. Marques AP, Kondo A. A fisioterapia na osteoartrose: Uma revisão da literatura. Rev Bras Reumatol 1998; 38(2): 83-90.

9. Bellamy NW, Buchanan WWGCH, Campbell JSLW. Validation study of Womac: A health status instrument for measuring clinically important patient relevant outcomes to antirheumatic drug therapy in patients with osteoarthritis of the hip and the knee. J Rheumatol 1988; 15: 1833-40.

10. Al-Zharani KS, Bakheit AMO. A study of the gait characteristics of patients with chronic osteoarthritis of the knee. Dis Rehab 2002; 24 (5): 275-80.

11. Camargos FFO, Lana DM, Dias RC, Dias JMD. Estudo da propriocepção e desempenho funcional em idosos com osteoartrite de joelhos. Rev Bras Fis 2004; 8 (1): 1-7.

12. Fransen M, Crosbie J. Edmond J. Reliability of gait measurements in people with osteoarthrits of the knee. Phys Ther 1997; 77 (9): 944-53.

13. Williams DA, Farrel MJ, Cunningham J, Gracely RH, Ambrose $\mathrm{K}$, Cupps T, et al. Knee pain and radiographic osteoarthritis interact in the prediction of levels of self-reported disability.

14. Van Baar M, Dekker J. Lemmens AM, Oostendorp RAB. Pain and disability in patients with osteoarthritis of hip or knee: the relationship with articular, kinesiological, and psychological characteristics. J Rheumatol 1998; 25 (1): 125-133.

15. McAlindon TE, Cooper C, Kirwan J, Dieppe P. Determinants of disability in osteoarthritis of the knee. Ann Rheum Dis 1993; 52: 258-62.

16. Creamer P, Lethbridge-Cejku M, Hochberg MC. Factors associated with functional impairment in symptomatic knee osteoarthritis. Rheumatol 2000; 39: 490-6.
17. Lamb SE, Guaralnik JM, Buchner DM, Ferrucci LM, Hochberg MC, Simonsick EM, et al. Factors that modify the association between knee pain and mobility limitation in older women: the Women's Health and Aging Study. Ann Rheum Dis 2000; 59: 331-7.

18. Ivanovith MF. Tradução e validação do questionário de qualidade de vida específico para a osteoartrose WOMAC (Western Ontario and McMaster Universities Osteoarthtis Index) para a língua portuguesa. Tese de Mestrado - Universidade Federal de São Paulo - Escola Paulista de Medicina; 2002.

19. Kellgren JH, Lawrence, JS. Radiological assessment of osteoarthrosis. Ann Rheum Dis 1957;16: 494-502.

20. Luepongsak N, Amint S, Krebs DE, McGibbon CD, Felson D. The contribution of type of daily activity to loading across the hip and knee joints in the elderly. Osteoarthritis and Cartilage 2002; 10 (5):353-59.

21. Gür H, Çakin N. Muscle mass, isokinetic torque, and functional capacity in women with osteoarthritis of the knee. Arch Phys Med Rehabil 2003; 84: 1534-41.

22. Topp R, Woolley S, Khuder S, Hornyak J, Bruss A. Predictors of four functional tasks in patients with osteoarthritis of the knee. Orthop Nurs 2000; 19 (5): 49-58.

23. DeVita P, Hortobágyi T. Obesity is not associated with increased knee joint torque and power during level walking. J Biomechanics 2003; 36: 1355-62.

24. Hurwitz DE, Sharma L, Andriacchi TP. Effect of knee pain on joint loading in patients with osteoartrhitis. Curr Opin Rheumatol 1999; 11 (5): 422-6.

25. Harrinson AL. The influence of pathology, pain balance and selfefficacy on function in women with osteoarthritis of the knee. Phys Ther 2004; 84: 822-31.

26. Salaffi F, Cavalieri F, Nolli M, Ferraccioloi G. Analysis of disability in knee osteoarthritis. Relationship with age and psychological variables but not with radiographic score. J Rheumatol 1991; 18 (10): 1581-6.

27. Stratford PW, Kennedy D, Pagura SM, Gollish JD. The relationship between self-report and performance-related measures: questioning the content validity of timed tests. Arthritis Rheum 2003; 49 (4): 535-40. 